\title{
DEZ ANOS DE EXPERIÊNCIA DO COMITÊ DE ÉTICA EM PESQUISA DA SECRETARIA DE SAÚDE DO DISTRITO FEDERAL, BRASIL
}

\author{
Maria Rita C. G. Novaes*, Dirce Guilhem* y Fernando Lolas** \\ Resumo: O objetivo deste artigo é relatar a experiência do Comitê de Ética em Pesquisa da Secretaria de Estado de Saúde \\ do Distrito Federal (CEP/SES/DF) Brasil, durante o período de 10 anos a partir de sua fundação. \\ Trata-se de uma avaliação descritiva e documental, na modalidade estudo de caso, utilizando-se a totalidade de projetos \\ protocolados no CEP/SES/DF (No 052/08) nesse período. \\ As pendências mais freqüentes dos projetos foram: termo de consentimento livre e esclarecido (30\%), folha de rosto (25\%), \\ metodologia (20\%), curriculum vitae (12\%), planilha de orçamento $(9 \%)$, outros $(4 \%)$. \\ $\mathrm{O}$ relato das atividades do CEP/SES/DF no período de 10 anos revelou, através de sua produtividade, a legitimidade do \\ processo de análise ética dos protocolos visando à proteçáo dos participantes da pesquisa.
}

Palavras chave: ética em pesquisa, comitês de ética, avaliação

\section{DIEZ AÑOS DE EXPERIENCIA DEL COMITÉ DE ÉTICA E INVESTIGACIÓN DE LA SECRETARÍA DE SALUD DEL DISTRITO FEDERAL, BRASIL}

Resumen: El objetivo de este artículo es relatar la experiencia del Comité de Ética en Investigación de la Secretaría de Estado de Salud del Distrito Federal (CEP/SES/DF), Brasil, durante un período de 10 años (1997-2007) a partir de su fundación. Se trata de una evaluación descriptiva y documental, en la modalidad estudio de caso, utilizándose la totalidad de proyectos protocolizados en el CEP/SES/DF (Número 052/08) en ese período.

Los conflictos más frecuentes fueron: término de consentimiento libre e informado (30\%), protocolo de investigación (25\%), metodología (20\%), currículum vitae (12\%), planilla de presupuesto (9\%), otros (4\%).

$\mathrm{El}$ informe de las actividades del CEP/SES/DF en 10 años reveló, a través de su productividad, la legitimidad del proceso de análisis ético de los protocolos, confirmando la protección de los participantes en la investigación.

Palabras clave: ética en investigación, comités de ética, valoración

\section{TEN YEARS OF EXPERIENCE WITH THE RESEARCH ETHICS COMMITTEE OF THE SECRETARY OF HEALTH, FEDERAL DISTRICT, BRAZIL}

Abstract: The objective of this article is to relate the experience of the Research Ethics Committee of the Secretary of Health, Federal District, Brazil (CEP/SES/DF) during 10 years (1997-2007) from its creation.

It deals with a descriptive and documentary evaluation, in the form of case studies, utilizing all projects subject to protocol in CEP/SES/DF (Number 052/08) during that period.

The most frequent conflicts were: terms of free and informed consent (30\%), identification page (25\%), methodology (20\%), curriculum vitae (12\%), budget (9\%), others (4\%).

The activity report of CEP/SES/DF in 10 years revealed, as a result of its productivity, legitimacy in the process of ethical analysis of protocols which confirms the protection of research participants.

Key words: ethics in investigation, ethics committees, validation

\footnotetext{
Coordenadora do Comitê de Ética em Pesquisa, Secretaria de Saúde do Distrito Federal, Brasil

Correspondencia: ritanovaes@ig.com.br

** Presidente do Foro Latinoamericano de Comités de Ética en Investigación em Salud (Flaceis), Brasil

*** Diretor do Centro Interdisciplinario de Estudios en Bioética, Universidad de Chile, Chile
} 


\section{Introdução}

No Brasil, a primeira regulamentação para pesquisas envolvendo seres humanos foi a Resolução $\mathrm{n}^{\circ} 01$ do Conselho Nacional de Saúde (CNS), divulgada no ano de 1988. Essa diretriz apontava para a necessidade de normatizar os aspectos éticos relacionados às pesquisas que incluíam participantes humanos. A resolução incluía aspectos relativos às pesquisas de novos recursos profiláticos, diagnósticos, terapêuticos e de reabilitaçáo; estudos clínicos em farmacologia, protocolos com crianças; pesquisas realizadas com órgãos, tecidos e seus derivados e, ainda, recomendava a criação de comitês internos nas instituiçóes de saúde(1).

Em 1996, o Tratado do Mercado Comum do Sul (Mercosul) elaborou a Resolução n ${ }^{\circ}$ 129/96, intitulada de Boas Práticas Clínicas. Esta resolução tem como alvo pesquisas na área de Farmacologia Clínica no que se relaciona aos seguintes aspectos: autorização, acompanhamento do estudo, responsabilidades dos pesquisadores e dos patrocinadores, requisitos éticos a serem seguidos e a necessidade de obtenção de informação pré-clínica e clínica para dar prosseguimento às investigaçôes(2).

Esses documentos deram origem à criação de alguns Comitês de Ética em Pesquisa (Cep) no Brasil, que estavam vinculados a hospitais, clínicas e serviços especializados. Esses comitês se distinguiam dos colóquios, institutos e outros lugares onde a ética é objeto de debates e ensino. Eles tinham a função de reunir, além de homens e mulheres de ciência, juristas e filósofos, até mesmo simples cidadãos capazes de contribuir com uma visão diferenciada para o exame dos fundamentos éticos que deveriam nortear as pesquisas com seres humanos. Porém, sua forma jurídica e composição não estava bem definidas e isso foi um elemento impeditivo para sua incorporação à prática de pesquisa $(3,4)$.

Como a Resolução 01/88 não obteve o resultado de normatizaçáo desejado entre os membros da comunidade científica, surgiu a necessidade de se elaborar um novo documento nacional que abarcasse de forma ampliada abordando os aspectos éticos requeridos na realização pesquisas. Foi criado um grupo de trabalho pelo Conselho Nacional de Saúde (CNS), que após ampla discussão com diferentes atores, entre eles: cientistas, gestores, representantes de instituiçôes de ensino e de pesquisa, e membros da sociedade, elaboraram a nova resoluçáo que foi divulgada em 1996.

A Resolução CSN 196/96, intitulada Diretrizes e Normas Regulamentadoras de Pesquisas envolvendo Seres Humanos, incorpora os princípios bioéticos da autonomia, beneficência, não-maleficência e justiça. No entanto, princípios adicionais estão contemplados, tais como: confidencialidade, privacidade, voluntariedade, equidade e não estigmatização(5). Além disso, “... um dos pontos-chave do documento é a ênfase no controle social, uma prerrogativa que ultrapassa as esferas técnica e científica, contribuindo para a efetivação do controle social no âmbito da ciência"(6:19). Esses requisitos demonstram a seriedade com que os aspectos éticos deverão ser enfrentados durante o processo de avaliação e acompanhamento ético das pesquisas.

A partir da Resolução 196/96, foi criado o Sistema Cep/Conep, um sistema nacional de avaliação ética das pesquisas envolvendo seres humanos, que compreende uma Comissão Nacional de Ética em Pesquisa (Conep) e Comitês de Ética em Pesquisa (Ceps) que estáo vinculados às instituições de ensino e pesquisa, hospitais, clínicas, organizaçóes não governamentais, secretarias de saúde, entre outras possibilidades. A Conep tem função consultiva, deliberativa, normativa e educativa e atua de forma conjunta com os Ceps. Constitui-se como um colegiado multiprofissional e transdisciplinar, que comporta por profissionais de destacada atuaçáo nas áreas da ética, saúde, pesquisa e com reconhecida atuação em áreas específicas do conhecimento científico. Estão representados, também, diferentes segmentos da sociedade civil organizada(4).

Com o rápido desenvolvimento científico e tecnológico, a reflexão ética sobre os protocolos efetuada a partir das diretrizes contidas na Resolução 196/96, necessitou ser ampliada. Foram elaboradas resoluçôes complementares para abarcar os novos desafios colocados pelas áreas temáticas especiais, como pode ser visualizado no Quadro 1. 
Quadro 1 - Resoluçôes publicadas pelo Conselho Nacional de Saúde, Ministério da Saúde, Brasil, utilizadas na avaliação ética de protocolos de pesquisa $(5,7,8)$.

\begin{tabular}{|l|l|l|}
\hline Ano & Resoluçáo & Observação \\
\hline 1996 & Resoluçáo CNS 196/96 & Diretrizes e Normas Regulamentadoras de Pesquisa Envolvendo Seres Humanos. \\
\hline 1997 & Resolução no 240/97 & Define representação de usuários nos CEPs e orienta a escolha. \\
\hline 1997 & Resoluçáo CNS 251/97 & $\begin{array}{l}\text { Norma complementar para a área temática especial de novos fármacos, vacinas e testes diagnósticos. } \\
\text { Delega aos CEPs a análise final dos projetos exclusivos dessa área, quando não forem enquadrados } \\
\text { em outras áreas especiais. }\end{array}$ \\
\hline 1999 & Resoluçáo CNS 292/99 & $\begin{array}{l}\text { Normas específicas para a aprovação de protocolos de pesquisa com cooperação estrangeira, mantendo } \\
\text { o requisito de aprovaçáo final pela CONEP, após aprovação do CEP. }\end{array}$ \\
\hline 2000 & Resolução CNS 303/00 & $\begin{array}{l}\text { Norma complementar para a área de Reprodução Humana, estabelecendo subáreas que devem ser } \\
\text { analisadas na Conep e delegando aos Ceps a análise de outros projetos da área temática. }\end{array}$ \\
\hline 2000 & Resolução CNS 304/00 & Norma complementar para a área de Pesquisas em Povos Indígenas. \\
\hline 2004 & Resoluçáo CNS 340/04 & $\begin{array}{l}\text { Diretrizes para Análise Ética e Tramitaçáo dos Projetos de Pesquisa da Área Temática Especial de } \\
\text { Genética Humana. }\end{array}$ \\
\hline 2005 & Resolução CNS 346/05 & Projetos multicêntricos. \\
\hline 2005 & Resolução CNS 347/05 & $\begin{array}{l}\text { Diretrizes para análise ética de projetos de pesquisa que envolva armazenamento de materiais ou uso } \\
\text { de materiais armazenados em pesquisas anteriores. }\end{array}$ \\
\hline 2007 & Resolução CNS 370/07 & Registro e credenciamento do Cep na Conep ou renovaçáes no registro do Cep. \\
\hline
\end{tabular}

O Comitê de Ética em Pesquisa em Seres Humanos da Secretaria de Estado de Saúde do Distrito Federal - CEP/SES/DF é uma instância colegiada multiprofissional e transdisciplinar, de natureza consultiva, deliberativa, normativa, educativa e independente, registrado na Comissão Nacional de Ética em Pesquisa (Conep) do Ministério da Saúde (MS) em 18 de junho de 1997(9). Após esse primeiro credenciamento, o comitê tem sido novamente recredenciado a cada três anos.

Na literatura brasileira, observam-se poucas referências relacionadas às atividades e produtividade de um Cep, considerando-se que o Brasil possui hoje cerca e 600 Ceps cadastrados junto à Conep(10-17). Avaliar a qualidade de atividades complementares àquelas desenvolvidas em um serviço de saúde é requisito importante para mostrar transparência sobre o trabalho desenvolvido e para fornecer à sociedade elementos que lhe permitam conhecer o desempenho de determinado grupo ou equipe. Proporciona, ainda, a oportunidade de identificar soluçóes para as dificuldades encontradas, propor mudanças e definir novas metas e desafios, de forma a qualificar as açóes desenvolvidas pelo comitê.

O objetivo deste trabalho é relatar a experiência do CEP/SES/DF, no período compreendido entre setembro de 1997 a dezembro de 2007, avaliando os seguintes aspectos: categoria e freqüência dos projetos submetidos a avaliação, especialidade predominante dos projetos, tempo requerido para aprovaçáo dos protocolos, entre outros parâmetros de análise registrados pelo comitê.

\section{Métodos}

Este é um estudo descritivo e documental, que utilizou como referência o estudo de caso. Nesse processo foi dimensionada a totalidade de projetos protocolados no CEP/SES/DF no período compreendido entre setembro de 1987 a dezembro de 2007. Foram analisadas as informaçóes contidas nos campos de preenchimento obrigatório de todas as folhas de rosto dos protocolos de pesquisa submetidos ao CEP.

A Folha de Rosto acompanha o projeto de pesquisa, é um documento fundamental para a formalização da inserção do protocolo no contexto do Sistema Cep/Conep. As informaçóes nela contidas são essenciais para compor o banco de dados sobre os projetos em tramitação no Brasil e são automaticamente incluídas no Sistema Nacional de Informaçōes sobre Ética em Pesquisas envolvendo Seres Humanos(18). Nela estão incluídos dados relacionados à pesquisa, à identificação do pesquisador e do dirigente da instituição onde a pesquisa será realizada, quais seráo os participantes incluídos no estudo, as possíveis fontes de financiamento, entre outros aspectos(18).

Os dados foram alocados em sistema informatizado e os resultados gerados em programa Excel, versão 2007.

\section{Resultados e discussáo}

Ao longo dos 10 anos de existência do CEP/SES/DF, foram submetidos à apreciação do comitê 1129 projetos de pesquisas, como pode ser verificado na Figura 1. 
Figura 1. Projetos submetidos ao CEP/SES/DF no período de setembro de 1997 a dezembro de 2007 (N=1129).

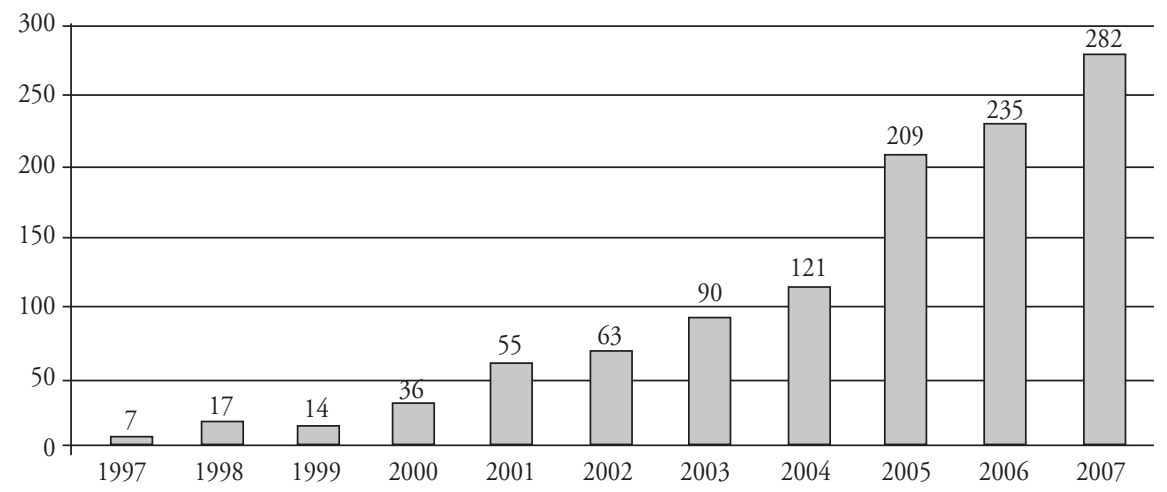

Nesse período houve aumento expressivo do número de protocolos avaliados. Alguns fatores foram determinantes para que isso ocorresse. $\mathrm{O}$ primeiro deles se deve ao caráter educativo efetuado pelo comitê junto aos pesquisadores, pois eles são orientados quanto à necessidade de submeter o projeto de pesquisa para a apreciação ética antes de se iniciar a sua execução. São alertados, ainda, que as revistas científicas indexadas exigem a carta de aprovação do projeto pelo Cep para a publicação dos artigos contendo o resultado de estudo que incluem seres humanos. Por outro lado, as agências nacionais e internacionais de financiamento de pesquisas exigem o parecer aprobatório pelo comitê para a liberação do recurso financeiro destinado ao projeto.

Outro fator relevante que contribuiu para o aumento do número de projetos submetidos ao CEP/SES/DF foi o aumento do número de cursos de graduação e pós-graduação, em especial na área de ciências da saúde, que estimulam a realização de estudos incluindo seres humanos. Dessa forma, é possível estabelecer vínculo importante entre o comitê, o pesquisador e as instituiçôes de ensino e pesquisa, pois o Cep atua como consultor no que se refere à utilizaçáo de condutas éticas, delimitadas a partir da Resolução 196/96. Torna-se, assim, um forte aliado do pesquisador na tentativa de assegurar a integridade dos participantes das pesquisas.

Além disso, começa a existir a consolidação de uma cultura ética de proteção dos direitos humanos no contexto das práticas científicas. Isso tem se refletido no processo de formação de jovens pesquisadores que incorporam os atributos éticos desde o início de suas carreiras $(19,20)$.
Os projetos submetidos para avaliação no período analisado foram 1129. Destes, 1021 protocolos foram aprovados (90,4\%); 17 projetos foram reprovados $(1,7 \%)$ por apresentarem questóes eticamente questionáveis; 84 protocolos $(7,4 \%)$ foram arquivados, retirados do processo porque os pesquisadores náo se manifestaram quanto às pendências apontadas pelo comitê ou, ainda, porque o próprio pesquisador solicitou o arquivamento do projeto devido à impossibilidade de realizá-lo por problemas operacionais. Foram excluídos da avaliação 05 projetos $(0,4 \%)$, referentes aos anos de 1997 e 1998, porque os dados referentes a estes projetos encontravam-se incompletos.

O baixo percentual de protocolos não aprovados pode ser compreendido como reflexo de açôes educativas promovidas pelo comitê. Após apreciação do projeto pelo relator e sua discussão nas reuniōes quinzenais do Cep, o pesquisador é imediatamente comunicado do parecer emitido. Se este for desfavorável, são apontadas as pendências que precisam ser atendidas, momento no qual o pesquisador é minuciosamente orientado pela coordenação do comitê para que possa trabalhar no sentido de adequar o projeto e submetê-lo novamente à apreciação ética.

Os protocolos de pesquisa foram classificados pela Conep em Grupos I, II e III, sendo que cada uma dessas classes abarca projetos de diferentes áreas e complexidade. No Grupo I estáo incluídos protocolos provenientes de áreas temáticas especiais como Genética humana; Reprodução humana; Novos equipamentos, insumos e dispositivos; Novos procedimentos; Populaçôes indígenas; Biosegurança e Pesquisas com Cooperação Estrangeira. No Grupo II estão relacionadas protocolos 
pertencentes a áreas temáticas como Novos Fármacos, Vacinas e Testes Diagnósticos. O Grupo III compreende projetos em que os temas das pesquisas não se enquadram em áreas temáticas especiais(8).

Dos projetos submetidos ao CEP/SES/DF, a grande maioria (937-83\%) pertencia ao Grupo III, seguidos por protocolos incluídos nos Grupos I e II, com freqüência de $12 \%$ e 5\%, respectivamente (Figura 2). O baixo percentual atribuído ao Grupo II deve-se, possivelmente, à quase total inexistência de patrocinadores brasileiros nessa área, ausência de instalaçôes adequadas para o desenvolvimento dos projetos, longa duração da pesquisa e a necessidade de recursos humanos especializados, nem sempre existentes nas instituições de atenção à saúde.
A Resolução 346/05 trata dos aspectos relativos à apreciação de projetos de pesquisa multicêntricos. Caracterizam-se como estudos multicêntricos as "pesquisas conduzidas em dois ou mais centros, sempre com um protocolo comum, mas como uma administraçáo central e um centro único para receber e processar os dados" (8:78). O comitê recebeu para apreciação cerca de $18,0 \%$ de projetos que estavam incluídos nessa categoria (Figura 3).

A Resolução 292/99 diz respeito às pesquisas patrocinadas e coordenadas do exterior ou que tenham algum tipo de participação estrangeira. Compreende-se como participação estrangeira, qualquer tipo de apoio financeiro, institucional ou pessoal - no caso de vínculo entre pesquisadores - que sejam provenientes de países

Figura 2. Áreas temáticas dos projetos analisados pelo CEP/SES/DF no período de setembro de 1997 a dezembro de 2007 (N=1129).

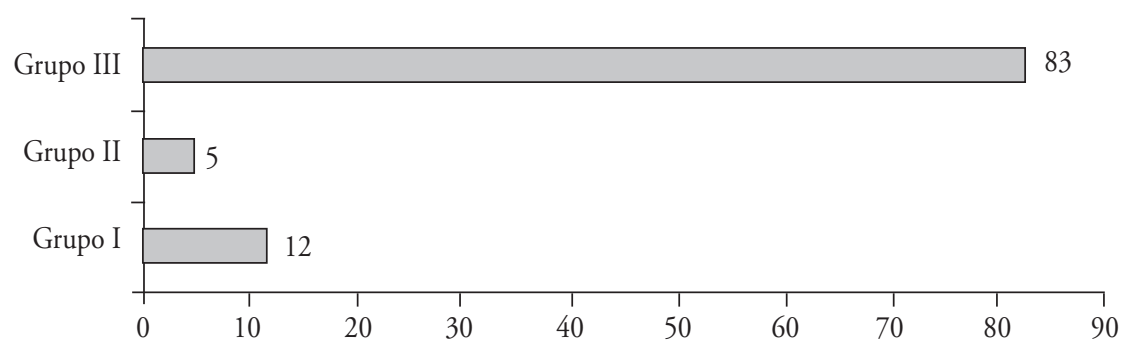

Nos dados analisados, não foi observada a predominância de pesquisas envolvendo indivíduos pertencentes a grupos especiais vulneráveis, susceptíveis a riscos e que seriam beneficiários dos resultados encontrados. Os grupos vulneráveis possuem resoluçóes complementares que regulamentam as açóes do Cep, dos pesquisadores e asseguram garantias aos sujeitos da pesquisa. Provavelmente devido a essas condiçôes especiais, esse grupo representou $17,0 \%$ dos projetos analisados pelo CEP.

Tabela 1. Classificação dos participantes das pesquisas dos projetos submetidos ao CEP/SES/DF no período de setembro de1997 a dezembro de 2007 (N=1129).

\begin{tabular}{|l|c|c|}
\hline Participantes das Pesquisas & $\begin{array}{c}\text { No. de } \\
\text { Projetos }\end{array}$ & $\%$ \\
\hline$<18$ anos & 169 & 15,0 \\
\hline Portador de deficiência mental & 8 & 1,0 \\
\hline Embrião ou feto & 1 & 0,01 \\
\hline $\begin{array}{l}\text { Relação de dependência (estudantes, } \\
\text { militares, presidiários, entre outros) }\end{array}$ & 9 & 1,0 \\
\hline Não se aplica a grupos especiais & 937 & 83,0 \\
\hline TOTAL & 1129 & 100,0 \\
\hline
\end{tabular}

estrangeiros. Tradicionalmente, incluem-se nesse grupo pesquisas vinculadas a empresas multinacionais, em sua maioria pertencente às áreas médicas e farmacêuticas. Grande parte das investigaçóes destina-se a realizar estudos comparativos com desenhos complexos que exigem a randomização de participantes, para avaliar novos fármacos (fases II, III e IV) e tecnologias, procedimentos ou aparelhos médico-hospitalares.

Nos protocolos de pesquisa submetidos ao CEP/SES/ $\mathrm{DF}$, a modalidade participação estrangeira representou $10 \%$ dos projetos avaliados no período de estudo. Nesse grupo, a maioria das investigaçóes era de estudos multicêntricos internacionais.

No que se refere aos patrocinadores dos estudos, $80 \%$ dos projetos apreciados pelo $\mathrm{CEP} / \mathrm{SES} / \mathrm{DF}$, não teve patrocínio estrangeiro. Os pesquisadores relataram que os projetos receberam apoio financeiro de instituições públicas brasileiras (agências de fomento governamentais), organismos internacionais, organizaçóes não governamentais, recursos provenientes do próprio 
pesquisador ou fornecidos pela instituição na qual estão inseridos.

A análise ética e a emissão do parecer inicial pelo Cep devem ser realizadas em um prazo máximo de 30 dias(5). O CEP/SES/DF tem atendido as recomendaçóes da Resoluçáo 196/96 e quando o protocolo de pesquisa apresenta alguma pendência apontada pelo comitê, o pesquisador tem um prazo de 60 dias para apresentar a resposta aos quesitos indicados. $\mathrm{Na}$ presença de pendências, o prazo de aprovação (ou não) pelo CEP/SES/DF variou de 30 a 60 dias, tendo havido diminuiçáo considerável do período de análise nos últimos anos.

As pendências mais freqüentes encontradas nos projetos avaliados pelo colegiado do Cep estão representadas na Figura 3. Referem-se à solicitação de reavaliação do TCLE (30\%); informaçôes incompletas na Folha de Rosto (25\%), Metodologia com ausência de informaçóes a respeito do tipo de estudo, amostragem, critérios de inclusão e exclusão, forma de alocação e análise dos dados (20\%); Planilha de Orçamento ausente ou incompleta (12\%); Curriculum Vitae de todos os pesquisadores citados: incompleto ou ausente (9\%); Outros Fatores (4\%). tólogos (2\%); outros (17\%). Na categoria "outros" enquadram-se os seguintes pesquisadores: arquiteto, antropólogo, biomédico, educador físico, administrador, advogado, bibliotecário, bombeiro, engenheiro civil, epidemiologista, jornalista, patologista, pedagogo, químico, sociólogo e microbiologista.

\section{Conclusão}

A responsabilidade de um comitê de ética em pesquisa está definida em diretrizes éticas internacionais, regulamentaçôes e legislação nacional. No caso brasileiro, existe sólida base regulamentar que ressalta a necessidade de revisão ética e científica das pesquisas que envolvem seres humanos. Os princípios éticos utilizados para nortear o processo de investigação são considerados universais, no entanto, a existência de documentos internacionais e de regulamentação nacional representa apenas o passo inicial para a adoção desses princípios.

Algumas questóes mostram-se mais relevantes para países em desenvolvimento e para as comunidades onde as pesquisas são realizadas. Cuidado especial dever ser direcionado para populações vulneráveis em cenários de desigualdade social e cultural. Sob essa

Figura 3. Pendências mais freqüentes nos projetos apreciados pelo CEP/SES/DF no período de setembro de 1997 a dezembro de 2007 $(\mathrm{N}=1129)$.

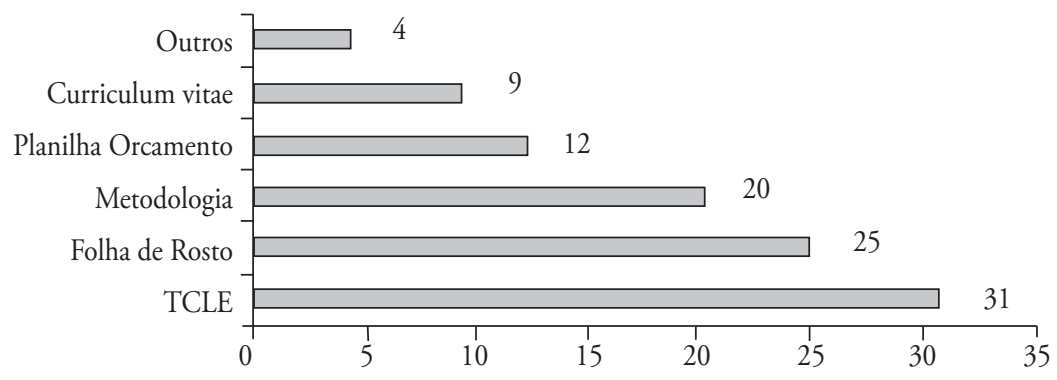

Os projetos apreciados pelo Cep no período do estudo mencionavam participação multiprofissional da equipe que conduzia a pesquisa. No que se refere à formação do pesquisador principal pôde-se perceber que eles eram provenientes de diferentes categorias profissionais da área da saúde, provavelmente devido à característica assistencialista presente nos serviços de atenção à saúde do Distrito Federal. Os coordenadores estavam assim distribuídos: médicos (35\%); enfermeiros (18\%); nutricionistas (7\%); psicólogos (7\%); biólogos (5\%): farmacêuticos (5\%); assistentes sociais (4\%); odon- ótica, a responsabilidade pela ética da prática científica inclui não apenas pesquisadores, membros de comitês, gestores e instituições envolvidas nesse processo, mas dizem respeito a toda a sociedade. Isso aponta para a importância do controle social das pesquisas envolvendo seres humanos.

O diagnóstico sobre as atividades desenvolvidas pelo CEP/SES/DF durante o período de 10 anos revelou o empenho do comitê para propiciar a análise ética das pesquisas submetidas à avaliação. Demonstrou que 
o comitê tem legitimidade para efetuar esse trabalho atuando como instrumento de valorização e estímulo para o desenvolvimento da produção científica no âmbito da SES/DF.

Essa avaliação diagnóstica se tornou possível pelo fato de que o comitê dispunha de arquivos desde o período de sua criação. Muito embora, a resolução prevê o arquivamento pelo período de cinco anos, os autores sugerem aos comitês que não descartem as informaçôes relativas aos protocolos apreciados sem antes efetuarem avaliação detalhada das atividades realizadas. Isso contribuirá para aprimorar o processo de trabalho e para definir estratégias que irão conferir credibilidade e transparência às atividades de um comitê de ética em pesquisa.

Os autores agradecem aos especializandos da Universidade de Brasília: Alexandre Alvares Martins, Herbenio Elias Pereira, Izabella de Vicente Maróstica, por terem colaborado na coleta de dados.

\section{Referências}

1. Brasil. Ministério da Saúde. Conselho Nacional de Saúde. Resolução nº 01/88. Normas de pesquisa em Saúde. Disponível em URL: http://conselho.saude.gov.br/resolucoes/1988/Reso01.doc

2. Mercosul. Grupo Mercado Comum. Resolução No 129/96. Boas práticas clínicas. Disponível em URL: http://www. ufrgs.br/bioetica/bpcmerco.htm

3. Brasil. Ministério da Saúde. Conselho Nacional de Saúde. Resolução nº 01/88. Normas de pesquisa em Saúde. Disponível em URL: http://conselho.saude.gov.br/resolucoes/1988/Reso01.doc

4. Troster EJ. Comitês de Bioética. Rev. Assoc. Med. Bras. 2000; 46(4): 296-297.

5. Brasil. Ministério da Saúde. Conselho Nacional de Saúde. Resolução 196/96. Diretrizes e normas regulamentadoras de pesquisas envolvendo seres humanos. Brasília: Ministério da Saúde; 1996.

6. Guilhem D, Diniz D. Ética na pesquisa no Brasil. In: Diniz D, Guilhem D, Schüklenk U, (eds.) Ética na pesquisa: experiência de treinamento em países sul-africanos. Brasília: Letras Livres/Editora UnB; 2005: 11-28.

7. Brasil. Ministério da Saúde. Resoluçōes do Conselho Nacional de Saúde: 240/97; 251/97; 251/97; 292/99; 301/00; 303/00; 204/00; 346/05; 347/05; 370/07. Disponível em URL: http://www.conselho.saude.gov.br/comissao/conep/relatorio. doc.

8. Brasil. Ministério da Saúde. Conselho Nacional de Saúde. Manual Operacional para Comitês de Ética em Pesquisa. Brasília: Ministério da Saúde, 4a edição; 2007.

9. Fepecs. Fundação de Ensino e Pesquisa em Ciências da Saúde. Comitê de Ética em Pesquisa da Secretaria de Estado de Saúde do Distrito Federal. Disponível em URL: http://www.fepecs.edu.br/pcomitedeetica.htm

10. Hardy EB, Bento SF, Osis MJD, Hebling EM. Comitês de Ética em Pesquisa: adequação à Resolução 196/96. Rev. Assoc. Med. Bras. 2004; 50(4): 457-462.

11. Novaes MRCG. O Comitê de Ética em Pesquisa. In: Guilhem D, Sorokin P, Queiroz W, Zicker F, (eds.) Ética em Pesquisa: compartilhando responsabilidades. Brasilia: Letras Livres; Editora UnB, 2008 (no prelo).

12. Barros SMO, Pinus J. Análise ética de protocolos de pesquisa: a experiência do Comitê de Ética em Pesquisa do Hospital Israelista Albert Einsteins (HIAE). Einstein (periódico on line) 2005; 1:4-7. Disponível em URL: http://www.einstein. $\mathrm{br} /$ revista/biblioteca/artigos/vol3/suplemento/Vol3_Supl_P4.pdf

13. A Experiência do Comitê de Ética em Pesquisa da Universidade Federal de Minas Gerais (COEP/UFMG) -1997/98. Disponível em URL: http://www.portalmedico.org.br/revista/bio2v6/expcometicaufmg.htm

14. A Experiência do Comitê de Ética em Pesquisa da Pontifícia Universidade Católica do Rio Grande do Sul, do Comitê de Bioética do Hospital São Lucas e da Faculdade de Medicina da PUCRS. Disponível em URL: http://www.portalmedico. org.br/revista/bio2v6/expcometicapucrs.htm

15. A Experiência dos Comitês de Ética no Hospital de Clínicas de Porto Alegre. Disponível em URL: http://www.cremal.org. $\mathrm{br} /$ revista/bio2v6/expcometicapa.htm

16. Comitê de Ética em Pesquisa: um parceiro no processo educativo. Disponível em URL: http://www.ghente.org/publicacoes/ moralidade/processo_educativo.pdf

17. Comitês de Ética em Pesquisa nas Universidades - o Desafio de Educar. Disponível em URL: http://www.abem-educmed. org.br/rbem/pdf/volume_25_2/editorial.pdf 
Dez anos de experiência do Comitê de Ética em Pesquisa - Maria Rita C. G. Novaes, Dirce Guilhem y Fernando Lolas

18. Brasil. Ministério da Saúde. Conselho Nacional de Saúde. Sistema nacional de informaçóes sobre ética em pesquisas envolvendo seres humanos - SISNEP. Brasília: CNS/ 2004. Disponível em URL: http://conselho.saude.gov.br/Web_ comissoes/conep/aquivos/sisnep/apresentacao.ppt.

19. Zicker F, (coord.) Guilhem D, Diniz D, (orgs.) Bioética e pesquisa com seres humanos: programas acadêmicos e de extensão. Brasília: Letras Livres; 2006.

20. Guilhem D, Zicker F. Ética na pesquisa em saúde: avanços e desafios. Brasilia: Letras Livres; Editora UnB; 2007.

Recibido: 6 de abril de 2008

Aceptado: 2 de junio de 2008 